\title{
Pharmacotherapy of diabetes and cancer risk
}

\author{
Kohei Kaku $\cdot$ Mitsuru Hashiramoto
}

Received: 22 August 2011/Published online: 23 September 2011

(C) The Japan Diabetes Society 2011

Lines of reports published recently analyzing the association between pioglitazone use and bladder cancer and the subsequent actions taken by regional regulatory agencies and international diabetes organizations rekindle a longrunning controversy over diabetes treatment and cancer risk.

An interim report of an ongoing cohort study, one of the representative papers published in the April issue of Diabetes Care, examined the association between pioglitazone therapy and the risk of bladder cancer in 193,099 diabetic patients in the Kaiser Permanente Northern California (KPNC) Diabetes Registry between 1997 and 2002. The patients were $\geq 40$ years of age and the group treated with pioglitazone comprised 30,173 patients. There were 90 cases of bladder cancer among pioglitazone users and 791 cases of bladder cancer among those who did not use pioglitazone. Overall, the short-term use of pioglitazone was not associated with a risk of bladder cancer [HR 1.2 (95\% CI 0.9-1.5)], but its use for more than 2 years was weakly associated with an increased risk (OR 1.4, 95\% CI 1.03-2.0) [1]. Surprisingly, France suspended the use of pioglitazone at the beginning of June 2011 and Germany has recommended that pioglitazone should not be started in new patients, based on a recently conducted retrospective cohort study by the French National Health Insurance Agency (Caisse National d'Assurance Maladie) which followed diabetic patients taking antidiabetic medicines between 2006 and 2009.

The US Food and Drug Administration (FDA) soon issued a Drug Safety Communication on June 15, 2011

K. Kaku $(\bowtie) \cdot$ M. Hashiramoto

Division of Diabetes, Metabolism, and Endocrinology,

Kawasaki Medical School, Kurashiki, Japan

e-mail: kka@med.kawasaki-m.ac.jp (updated on August 5) regarding the ongoing safety review of pioglitazone and the increased risk of bladder cancer [2]. Based on a review of the data from the KPNC epidemiological study, FDA stated that the long-term use of pioglitazone $(>1$ year) may be associated with an increased risk of bladder cancer. The FDA acknowledged that the finding was still of nominal statistical significance, and is advising health-care professionals not to use it in patients with active bladder cancer and to prescribe the drug with caution for patients with a prior history of bladder cancer, weighing the benefits of blood glucose control against the unknown risks of cancer recurrence. One month after the FDA's communication, the European Medicines Agency (EMA) issued a recommendation and the EMA's Committee for Medicinal Products for Human Use concluded that the benefits of pioglitzone outweigh its risks in a limited population of type 2 diabetes patients, despite the small risk of bladder cancer with this drug. The Committee has made recommendations to reduce the risk of bladder cancer in patients taking the medicines [3].

The series of events mentioned above reminded us of a recent outbreak of controversy related to a possible association between the long-acting insulin analog glargine and an increased risk of cancer. On June 2009, the diabetes community was suddenly shaken with a story relevant to patients with diabetes treated with insulin, when four registry studies that analyzed the association of insulin glargine with the risk of cancer were published in Diabetologia. The American Diabetes Association (ADA) immediately released a statement noting that "findings from these research papers are conflicting and inconclusive, and the ADA cautions against over-reaction and advises patients using insulin not to stop taking it until more information is available." Regulatory agencies such as the EMA and FDA, international organizations such as the International Diabetes Federation (IDF), 
etc., as well as most national diabetes organizations except for the Australian Diabetes Society subsequently released similar statements. This prompted a broad discussion of the safety of insulin versus insulin sensitizers in general and insulin analogs in particular, and the validity of the Diabetologia story was criticized from a variety of angles.

We have now reached the point again where we need to have another serious look at the highly complex questions concerning diabetes treatment and cancer risk. First of all, we must acknowledge the fact that cancer and diabetes develop within the same individual more frequently than would be expected. Based on the recent meta-analysis, some cancers develop more commonly (approximately twofold or higher for cancers of the liver, pancreas, and endometrium; 1.2- to 1.5-fold for cancers of the colon/ rectum, breast, and bladder) in patients with type 2 diabetes [4]. Nevertheless, whether diabetes and cancer are directly associated due to hyperglycemia, whether cancer risk is increased by the presence and duration of the diabetes itself, the exact roles (if any) of insulin resistance and hyperinsulinemia in the development of and/or association with cancer, and whether cancer and diabetes are indirectly associated due to common modifiable risk factors such as obesity, dietary habit, and physical inactivity are all still open questions. Fortunately, vigorous discussions of these issues have taken place since the insulin glargine story broke, and these are comprehensively reviewed in wellwritten lengthy statements like the Consensus Report by the American Diabetes Association and the American Cancer Society [5]. Thereby, we will limit ourselves here to stressing one of the most clinically relevant questions concerning the pharmacotherapy of diabetes and cancer risk.

We are very much aware of the fact that prospective large population-based studies are required to adequately address this issue- a comparison of the incidence of specific cancers between diabetic individuals with or without high circulating insulin levels and nondiabetic individuals with or without high circulating insulin levels. Unfortunately, a prospective clinical trial like this would be arguably unfeasible due to a range of practical difficulties and limitations. First, most diabetic patients are treated with more than one antihyperglycemic medication. Since all classes of antidiabetic drugs achieve glucose lowering by different mechanisms, it is extremely difficult to detect an independent association between a specific medication and cancer risk. The number of observational studies in humans which suggest that treatment with metformin is not associated with an increased risk of cancer, or rather decreases the risk compared with other glucose-lowering therapies, is growing. On the other hand, metformin is most typically prescribed to patients at an early stage of diabetes as the first-line drug; it is not usually given to elderly patients with severe hepatic and renal dysfunctions. Thus, this approach to metformin therapy may itself represent an obstacle to the proper and ethical design of the studies required. Secondary, type 2 diabetes is inherently progressive, leading to a variety of modifications to the associated pharmacotherapy over time. This adds extra complexity when designing studies to analyze the longterm outcome, and it also leads to ethical barriers against studies of this kind. Furthermore, some diabetes medications, such as thiazolidinediones, insulin analogs, and incretin-related drugs, have only recently come on the market. Thus, assessing the associations of these new agents with the risk of cancer in such studies would be rather difficult, as long-term observations would be needed to detect associations.

Confronted with the above-mentioned difficulties and limitations, it is unlikely that we will be able to fully address and clearly establish the effect of a specific diabetes medication on cancer risk, particularly at specific cancer sites, with prospective randomized controlled clinical studies. At this stage, what we can do is to wait for the accumulation of solid scientific information regarding the association of cancer risk with the use of individual antidiabetic medications. For the time being, we should not use cancer risk as a primary decision-making factor when choosing antidiabetic drugs for the average patient. We should maintain appropriate confidence in (while maintaining a sufficiently critical approach to) the safety and efficacy of the antihyperglycemic medications presently on the market, so that we do not impair the quality of the day-to-day control of blood glucose and lose sight of the objective of diabetes treatment: to improve the general quality of life and (in the long term) to reduce diabetes complications.

\section{References}

1. Lewis JD, Ferrara A, Peng T, et al. Risk of bladder cancer among diabetic patients treated with pioglitazone: interim report of a longitudinal cohort study. Diabetes Care. 2011;34:916-22.

2. FDA (2011) Update to ongoing safety review of Actos (pioglitazone) and increased risk of bladder cancer (FDA Drug Safety Communication). http://www.fda.gov/Drugs/DrugSafety/ucm259150. Accessed 17 Sept 2011.

3. European Medicines Agency (2011) European Medicines Agency recommends new contra-indications and warnings for pioglitazone to reduce small increased risk of bladder cancer. http://www.ema. europa.eu/ema/index.jsp?curl=pages/medicines/human/public_health_ alerts/2011/07/human_pha_detail_000033.jsp\&murl=menus/medicines/ medicines.jsp\&mid=WC0b01ac058001d126. Accessed 17 Sept 2011.

4. Vigneri P, Frasca F, Sciacca L, et al. Diabetes and cancer. Endocr Relat Cancer. 2009;16:1103-23.

5. Giovannucci E, Harlan DM, Archer MC, et al. Diabetes and cancer: a consensus report. Diabetes Care. 2010;33:1674-85. 